\title{
Erratum to: A Mobile Measurement System for the Coupled Monitoring of Atmospheric and Soil Parameters
}

\author{
A. V. Bazarov ${ }^{a *}$, N. B. Badmaev ${ }^{b, c}$, S. A. Kurakov ${ }^{d, e}$, \\ and B.-M. N. Gonchikov ${ }^{b}$ \\ ${ }^{a}$ Institute of Physical Materials Science, Siberian Branch, Russian Academy of Sciences, \\ ul. Sakh'yanovoi 6, Ulan-Ude, 670047 Russia \\ ${ }^{b}$ Institute of General and Experimental Biology, Siberian Branch, Russian Academy of Sciences, \\ ul. Sakh'yanovoi 6, Ulan-Ude, 670047 Russia \\ ${ }^{c}$ Buryat State University, ul. Smolina 24a, Ulan-Ude, 670047 Russia \\ ${ }^{d}$ Institute of Monitoring of Climatic and Ecological Systems, Siberian Branch, Russian Academy \\ of Sciences, pr. Akademicheskii 10/3, Tomsk, 634055 Russia \\ ${ }^{e}$ Tomsk State University of Control Systems and Radio-electronics, pr. Lenina 40, \\ Tomsk 634055 Russia \\ *e-mail: alebazaro@gmail.com \\ Received June 27, 2016
}

DOI: $10.3103 / \mathrm{S} 1068373918110110$

In this article the true affiliation is

\author{
A. V. Bazarov ${ }^{a *}$, N. B. Badmaev ${ }^{b, c}$, S. A. Kurakov ${ }^{d, e}$, \\ and B.-M. N. Gonchikov ${ }^{b}$ \\ ${ }^{a}$ Institute of Physical Materials Science, Siberian Branch, Russian Academy of Sciences, \\ ul. Sakh'yanovoi 6, Ulan-Ude, 670047 Russia \\ ${ }^{b}$ Institute of General and Experimental Biology, Siberian Branch, Russian Academy of Sciences, \\ ul. Sakh'yanovoi 6, Ulan-Ude, 670047 Russia \\ ${ }^{c}$ Buryat State University, ul. Smolina 24a, Ulan-Ude, 670047 Russia \\ ${ }^{d}$ Institute of Monitoring of Climatic and Ecological Systems, Siberian Branch, Russian Academy \\ of Sciences, pr. Akademicheskii 10/3, Tomsk, 634055 Russia \\ ${ }^{e}$ Tomsk State University of Control Systems and Radio-electronics, pr. Lenina 40, \\ Tomsk 634055 Russia \\ *e-mail:alebazaro@gmail.com \\ Received June 27, 2016
}

In this article techical mistakes were found. Below the correct variant of the table on page 273 is provided. 
Technical characteristics of the system

\begin{tabular}{|c|c|c|c|}
\hline $\begin{array}{l}\text { Number } \\
\text { in Fig. } 1\end{array}$ & Component & Range & Error \\
\hline 1 & Mast-tripod & $2 \mathrm{~m}$ & $\pm 0.3^{\circ} \mathrm{C}$ \\
\hline 2 & $\begin{array}{l}\text { 1-and 2-m air temperature and air humidity } \\
\text { sensor }\end{array}$ & $\begin{array}{c}-55 \ldots 50^{\circ} \mathrm{C} \\
0 \ldots 100 \%\end{array}$ & $\begin{array}{l} \pm 3.5 \% \\
\pm 5 \%\end{array}$ \\
\hline 3 & Sensor of wind speed and direction & $\begin{array}{c}0.9 \ldots 78 \mathrm{~m} / \mathrm{s} \\
0 \ldots 360^{\circ}\end{array}$ & $\pm 7 \%$ \\
\hline 4 & Snow depth sensor & $0 \ldots 1 \mathrm{~m}$ & $\pm 0.025 \mathrm{~m}$ \\
\hline 5 & Liquid precipitation sensor at the $2-\mathrm{m}$ mast & $0 \ldots 1000 \mathrm{~mm} /$ hour & $\pm 5 \%$ \\
\hline 6 & $\begin{array}{l}\text { Soil temperature profile sonde, depth }(\mathrm{m}): 0.15 \text {; } \\
0.2 ; 0.25 ; 0.3 ; 0.35 ; 0.4 ; 0.45 ; 0.5 ; 0.55 ; 0.6 \\
0.8 ; 1.0 ; 1.2 ; 1.6 ; 2.4 ; 3.2\end{array}$ & $-55 \ldots 50^{\circ} \mathrm{C}$ & $\pm 0.1^{\circ} \mathrm{C}$ \\
\hline 7 & Soil moisture sensor & $0 \ldots 100 \%$ & $\pm 2 \%$ (within $0 \ldots 70 \%$ ) \\
\hline 8 & Water conductivity sensor & $10-2 \times 10^{3} \mu \mathrm{S} / \mathrm{cm}$ & $\pm 20 \%$ \\
\hline 9 & $\begin{array}{l}\text { Case with control electronics and air pressure } \\
\text { sensor }\end{array}$ & $500-1150 \mathrm{hPa}$ & $\pm 10 \mathrm{hPa}$ \\
\hline
\end{tabular}

The original article can be found online at https://doi.org/10.3103/S106837391804009X. 